\title{
De la Volatilité des Salaires a la Croissance Excluante Dans le Mercosur 1991-2003: une Etude Statistique
}

Alexis Saludjian ${ }^{*}$

Résumé: L'objet de cet article vise à donner des arguments empiriques à la thèse de la croissance excluante qui aurait eu lieu en Amérique Latine durant le décennie 1990s. Selon cette thèse la croissance, malgré sa volatilité, n'a pas bénéficié de manière homogène dans la population du pays. Les salariés ont notamment été particulièrement touchés par la volatilité de la croissance et exclus de la croissance économique en terme de PIB réel. A partir d'une méthodologie développée par D. Rodrik, et d'une étude statistique originale, nous obtenons un guide illustratif sur l'expérience, en période de volatilité macro-économique, de ce régime de croissance excluant dans les pays du Cône Sud-Américain durant les années 1990.

Mots-clés: Regime de Croissance Excluant, Volatilité des Salaires, Volatilité Macro-Economique, Etude Statistique, Mercosur.

Abstract: The article aims at giving new empirical evidence of the excluding growth regime that happened in Latin America during the 1990s. Indeed, in spite of macroeconomic volatility, some economists suggest that growth did not benefit in an homogeneous way to the population. The wage earners have been particularly concerned by the macroeconomic volatility and excluded from the benefits of economic growth. Through a statistical study based on a methodology developed by D. Rodrik, we infer an illustrative guide of the excluding growth regime that has taken place in the 1990s in the Southern Cone Latin-American countries.

Keywords: Excluding Growth Regime, Wage Volatility, Macroeconomic Volatility, Statistical Study, Mercosur

\section{Introduction}

Cet article évalue les effets de la volatilité macro-économique sur le marché du travail. L'accent sera mis sur les salaires à travers une étude originale inspirée de travaux de D. Rodrik. Le travail représente la source essentielle des revenus des groupes de classe moyenne ou de bas salaires dans les régions urbaines. Cet élément a été déstabilisé et attaqué par une

Lauréat de la bourse Lavoisier 2004 du Ministère des Affaires étarngères. Docteur en Sciences Economiques, Université de Paris 13-Villetaneuse, UFR Sciences Economiques et Gestion. E-mail: saludjian@seg.univ-paris13.fr, alex_saludj@yahoo.fr. 
forme de production basée sur certains pôles modernes qui nécessite peu de main d'œuvre. Ce type d'emploi dans les secteurs à haute productivité et technologie n'a pas été générateur d'emploi en grand nombre et ce sont plutôt les secteurs à faible productivité qui ont employé la majorité de la population des classes sociales moyenne et pauvre .

L'objet de ce travail vise à donner des arguments empiriques à la thèse de la croissance excluante qui aurait eu lieu en Amérique Latine durant le décennie 1990s. Selon cette thèse la forte croissance n'a pas bénéficié de manière homogène dans la population du pays. Les salariés ont notamment été particulièrement touchés par la volatilité de la croissance et exclus de la croissance économique du PIB réel.

\section{La relation volatilité du PIB / volatilité des salaires:}

\subsection{Le travail et les salaires en Argentine et au Brésil depuis le Régionalisme Ouvert:}

Les graphiques suivants présentent l'évolution des niveaux de salaires réels moyens au Brésil et en Argentine?

Les données mensuelles des salaires réel moyen dans l'industrie argentine sont calculées par la CEPAL-Buenos-Aires (Graphique suivant).

\section{Graphique 1}

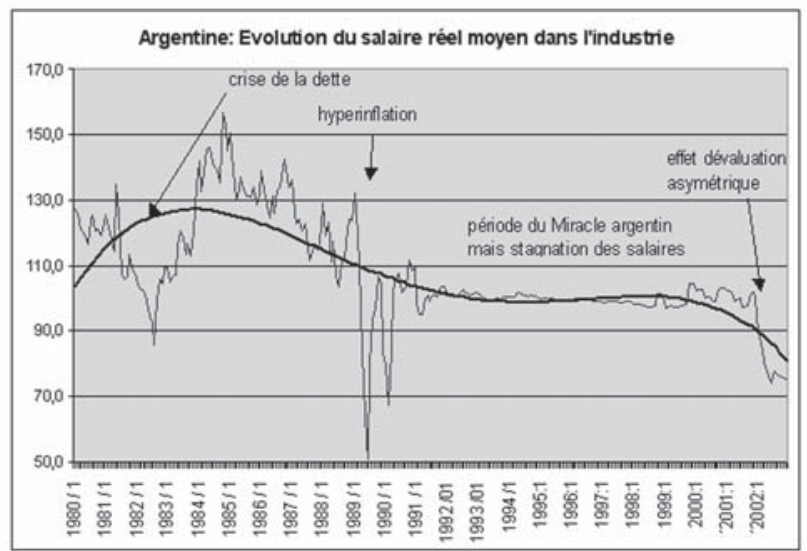

Source: CEPAL, 2003, Bureau de Buenos-Aires.

Note: Données mensuelles (1980/1: Janvier 1980). Définition du salaire réel moyen dans l'industrie d'après la CEPAL. Bureau de Buenos-Aires: "Correspond au salaire moyen normal et permanent défini comme la somme des salaires de bases (en incluant les heures supplémentaires et les primes). Valeurs avec saisonalité (mois dété soit décembre-février). Séries non corrigées pour la stationarité. De plus, les salaires réels se définissent par le montant des salaires nominaux mensuels déflatés par la moyenne IIPC du mois suivant comme estimateur des prix à la fin du mois". La courbe en noir (trait gras) correspond à une courbe de moyenne sur la période. 
La courbe de l'évolution des salaires réels moyens mensuels en Argentine amènent plusieurs remarques. On remarque des périodes extrêmement instables durant les crises hyperinflationnistes. L'élément qui retient notre attention est la stabilité des niveaux de salaires réels moyens à partir des plans de stabilisation macro-économique. En effet, durant la convertibilité argentine et malgré la volatilité du taux de croissance du PIB réel, les salaires argentins sont restés au même niveau. Cette remarque peut paraitre positive si la période considérée était caractérisée par une croissance faible ou nulle mais durant une part importante de cet épisode de la convertibilité, l'Argentine a connu des taux de croissance très élevés et ce, à de nombreuses reprises. Or les salaires n'ont absolument pas bénéficié des périodes de forte croissance ${ }^{3}$. Certains économistes noteront également que les salaires n'ont pas baissé pendant les baisses du taux de croissance après l'effet Tequila notamment soulignant ainsi le caractère rigide des salaires à la baisse comme à la hausse du à l'insuffisante flexibilisation du marché du travail, du poids trop important des syndicats et des fonctionnaires.

Les phases de croissance forte du PIB en 1991-1994 puis celle de 1996-1997 mais surtout l'accroissement de la productivité ont été déconnectées de l'évolution des salaires réels soulignant ainsi une des caractéristiques fondamentales de ce régime de croissance financiarisé .

Le partage des fruits de la croissance de ce régime à dominante financière a été inégal et excluant (voir tableaux 1 et 2).

\footnotetext{
${ }^{1}$ Voir (Salama, 1996 et 1999), (Katz, 2001), (Stallings et Peres, 2001), (Pizarro, 2001).

${ }^{2}$ Comme le constatent nombre de chercheurs spécialisées sur l'étude des niveaux de salaires dans ces pays (notamment [Wood, 1997, p. 40 et 55]) ces données sont relativement difficiles à mobiliser surtout en séries longues et homogènes. Cela est d'autant plus le cas pour des petits pays comme l'Uruguay et le Paraguay aussi nous centrerons nous sur les cas de l'Argentine et du Brésil. Les deux autres pays seront traités en fonction de la disponibilités des données. Pour le Brésil, l'étude en données mensuelles sur les deux dernières décennies sont disponibles (Ipeadata, 2003) mais ces chiffres sont des données brutes et non-désaisonnalisées. Une telle analyse amène à développer des considérations qui dépassent le cadre de ce travail, aussi nous présentons des données trimestrielles et annuelles au Brésil et en Argentine sur la décennie 1990. Dans le cas de l'Argentine, ces problèmes existent également mais nous disposons d'une série mensuelle déflatée et homogénéisée réalisée par la CEPAL-Buenos-Aires qui est éclairante. ${ }^{3}$ Ce constat est également fait par (Stallings et Weller, 2001, p. 190) qui soulignent que durant les années 1990 "l'Argentine a été l'exception à la règle, car en dépit des forts taux de croissance, aucune amélioration du niveau des salaires n'a été enregistrée".

${ }^{4}$ Voir Katz, Stumpo (2001), Moltimore et alii (2001), Frenkel et alii (2002).
} 


\section{Tableau 1}

Taux de croissance de la production industrielle, l'emploi et la productivité du travail dans le Mercosur et Etats-Unis dans le secteur manufacturiers, 1970-1996 (\%)

\begin{tabular}{|l|c|c|c|c|cc|}
\hline \multirow{2}{*}{ Pays } & \multicolumn{2}{|l|}{ Production industrielle } & \multicolumn{2}{c|}{ emploi } & \multicolumn{2}{c|}{ Productivité du travail } \\
\cline { 2 - 7 } & $1970-1996$ & $1990-1996$ & $1970-1996$ & $1990-1996$ & $1970-1996$ & $1990-1996$ \\
\hline Argentine & 1,18 & 4,87 & $-2,62$ & $-3,15$ & 3,80 & 8,02 \\
\hline Brésil & 2,81 & 2,26 & 0,95 & $-6,41$ & 1,86 & 8,67 \\
\hline Uruguay & 0,61 & $-1,46$ & 0,37 & $-8,58$ & 0,24 & 7,12 \\
\hline Etats-Unis & 2,39 & 5,04 & 0,35 & 0,30 & 2,04 & 4,74 \\
\hline
\end{tabular}

Source: (KA TZ, 2001, p.60). Calculs réalisés sur la base du Programme d'A nalyse des Dynamiques Industrielles (PADI) de la CEPAL.

Tableau 2

Productivité du travail industriel en par rapport au celle du secteur manufacturier des Etats-Unis, 1970-1996

\begin{tabular}{|c|c|c|c|c|}
\hline Pays & 1970 & 1980 & 1990 & 1996 \\
\hline Argentine & 0,42 & 0,41 & 0,55 & 0,67 \\
\hline Brésil & 0,28 & 0,26 & 0,29 & 0,37 \\
\hline Uruguay & 0,35 & 0,22 & 0,20 & 0,22 \\
\hline
\end{tabular}

Source (Katz, 2001, p. 60)

Note: Etats-Unis $=1,00$ en 1970

Si l'évolution de la productivité du travail a connu dans les années 1990 une croissance remarquable dans l'ensemble des pays du Mercosur, celle-ci n'en reste pas moins relativement éloingée du niveau des Etats-Unis. Alors que la production industrielle a augmenté en Argentine et au Brésil (par rapport aux niveaux faibles des années 1980), le taux de croissance de l'emploi indique des chiffres négatifs très élevés avec $-3,15 \%$ et $-6,41 \%$ en Argentine et au Brésil respectivement. Ces chiffres sont à mettre en parallèle avec les taux de croissance élevés des années 1990 et permettent d'avoir la situation de croissance forte mais de réduction de l'emploi, fondant ainsi le concept de croissance exluante.

Durant la décennie 1990, les évolutions des taux de salaires réels (annuel ou en moyenne sur 4 années/semestres mobiles) mais aussi de sa volatilité apportent des éléments intéressants qui confirment les trajectoires différentes du Brésil et de l'Argentine ${ }^{5}$.

\footnotetext{
${ }^{5}$ Voir (Saludjian, 2004b).
} 


\section{Graphique 2}

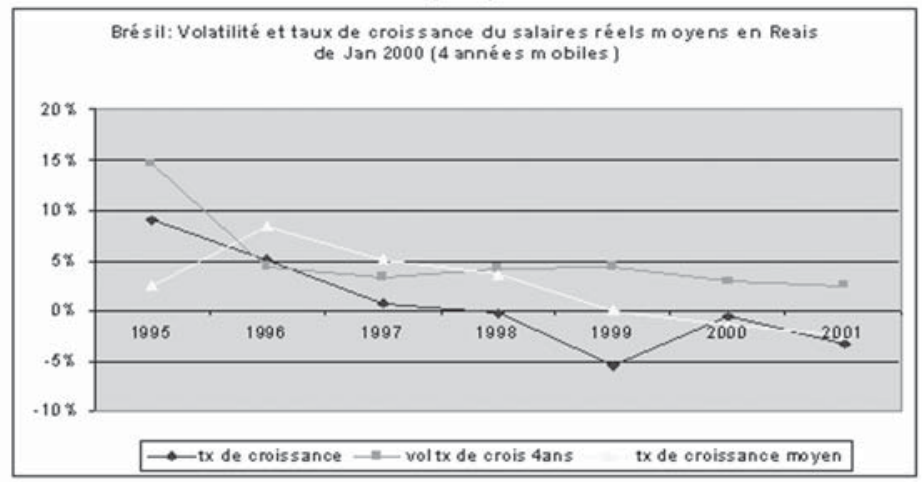

Source: IPEA-MTE, 2003.

Note: Les salaires correspondent à des salaires moyens dans toutes les régions métropolitaines.

\section{Graphique 3}

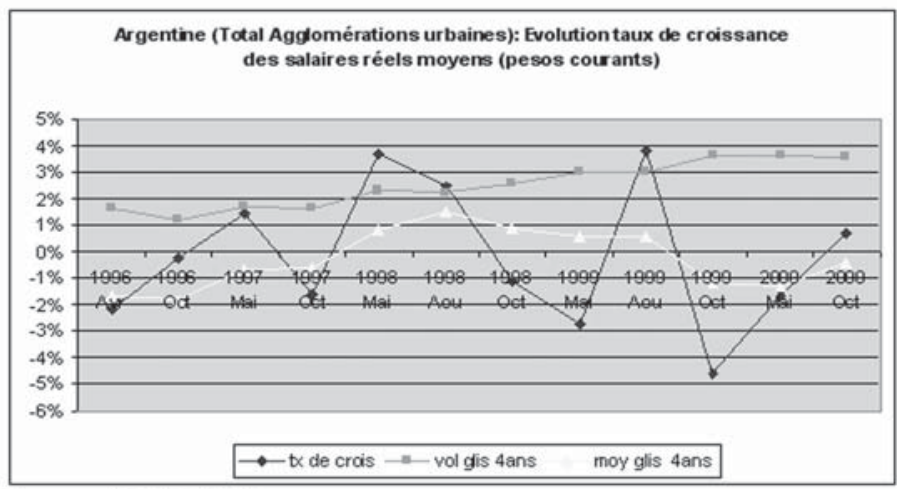

Source: INDEC, 2003.

Note: Salaire moyen en pesos courants (pendant la convertibilité)

Les graphiques en données annuelles et semestrielles de la volatilité et du taux de croissance moyen des salaires réels confirment la divergence de tendances des taux de croissance du PIB réel enregistrées dans (Saludjian, 2004b). Au Brésil la tendance est plutôt à une faible volatilité mais avec une faible croissance des salaires réels alors qu'en Argentine la volatilité est élevée et les taux de croissance ont été faibles ou négatifs. 
Nous allons pouvoir étudier les évolutions des taux de croissance du PIB et du salaire réel moyen en les mettant en regard dans un même graphique.

\section{Graphique 4}

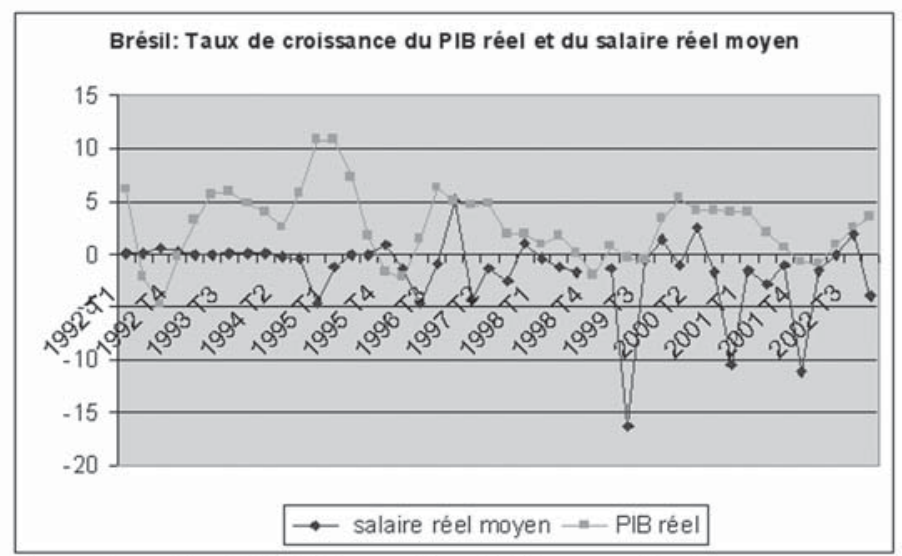

Source: I peadata, 2003. Salaires: même définition que précedemment.

\section{Graphique 5}

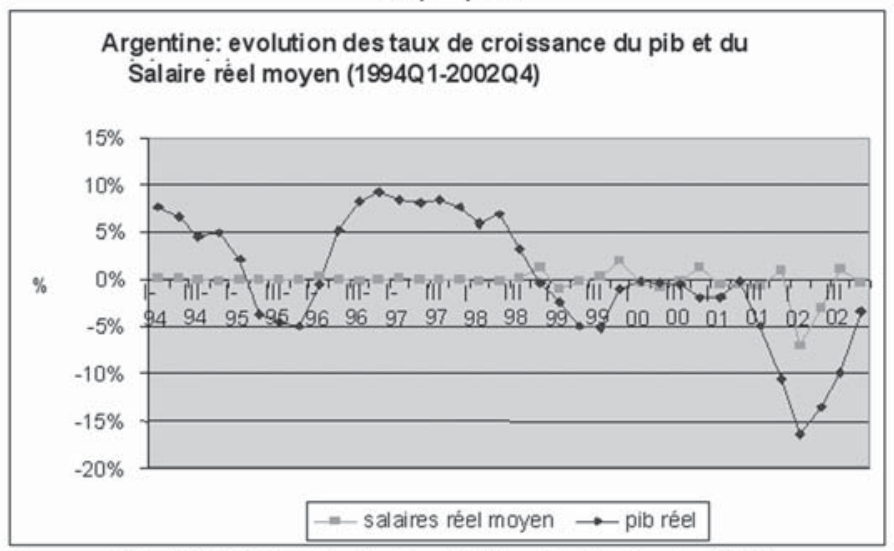

Sources: PIB: INDEC, Dirección Nacional de Cuentas Nacionales, 2003 Salaires: CEPAL, 2003, Bureau de Buenos-Aires. 
Ces données seront mobilisées dans la section suivante qui visera à décomposer la volatilité du salaire réel moyen. Elles montrent des évolutions différentes en Argentine (blocage des salaires malgré croissance élevée) et au Brésil.

Avant de procéder à l'analyse de la décomposition de la volatilité des salaires proposée par D. Rodrik, la taille de l'échantillon permet de mener un test de Causalité entre le taux de croissance du PIB réel et celui des salaires (en Argentine et au Brésil).

\section{Tableau 3}

Tests de causalité: volatilité macroéconomique et Volatilité

des taux de salaire réel moyen

\begin{tabular}{|l|c|c|c|c|}
\hline & $\begin{array}{c}\text { Nombre de } \\
\text { retards (2) }\end{array}$ & $\begin{array}{c}\text { Nombre } \\
\text { d'observations }\end{array}$ & $\begin{array}{c}\text { Txwage = } \\
\text { txpib }\end{array}$ & txpib ==> Txwage \\
\hline $\begin{array}{l}\text { Argentine } \\
\text { 1994Q1-2002Q4 }\end{array}$ & 2 & 34 & $\begin{array}{c}\text { NON } \\
0.675\end{array}$ & $\begin{array}{c}\text { OUI } \\
3.270^{* *}\end{array}$ \\
\hline $\begin{array}{l}\text { Brésil (1) } \\
\text { 1988M1-2002M12 }\end{array}$ & 4 & 229 & $\begin{array}{c}\text { OUI } \\
22.533^{*}\end{array}$ & $\begin{array}{c}\text { oUI } \\
4.082^{*}\end{array}$ \\
\hline
\end{tabular}

Source: Calculs de l'auteur. Logiciel Eviews

Notes: * signific atif au seuil de $1 \%$; "* significatif au seuil de $5 \%$;

(1) données mensuelles: IPEAdata 2003 taux de croissance à partir du PIB exprimé en prix de marché en base 100 .

(2) Comme précédemment, nous fixons un retard plus court dans le cas de l'Argentine en raison du niveau plus élevé de la volatilité dans ce pays.

Notons que dans ce type de test, le niveau de la volatilité du PIB importe peu puisque l'on s'intéresse au sens de la relation entre l'évolution de ces deux variables.

En Argentine, les salaires étant bloqués sur une longue période, il est normal qu'ils n'influencent pas la variation du PIB réel et que le test de Granger soit négatif dans ce sens. Ce résultat constitue une réfutation empirique pour le Mercosur de l'argument néoclassique qui considère que le maintien du niveau (relativement bas des salaires) ont une influence positive sur la croissance ${ }^{6}$. Cet argument libéral a pour objectif de souligner la nécessité de flexibiliser le marché du travail et de briser la rigidité des salaires à la baisse $^{7}$. Dans le cas de l'Argentine de 1994 à 2002, la causalité est au contraire vérifiée dans le sens inverse : la variation du PIB "cause au sens de Granger" (c'est-à-dire permet de mieux expliquer) la variation des salaires.

\footnotetext{
${ }^{6}$ Voir notamment les articles concernant la nécessaire flexibilisation du marché du travail dans l'ouvrage du "post-consensus de Washington": (Williamson J. et P-P Kuczynski (Coord.)), 2003.

7 Malgréson intérêt, nous ne pouvons-dans le cadre de cet article-nous attarder sur la vérification de l'argument keynésien qui voit dans l'évolution des salaires (via la demande effective) un moyen de dynamiser la croissance économique.
} 
Dans le cas brésilien, ce test montre la forte relation de type circulaire et autoentretenue entre variation du PIB et variation des salaires sur la période considérée.

Ces résultats donnent une première idée des liens entre variations macro-économiques et salaires.

\subsection{Analyse et décomposition de la volatilité des salaires réels de D. Rodrik}

\subsubsection{La logique et l'expression de la décomposition}

La décomposition proposée par $\mathrm{D}$. Rodrik a pour objectif de mieux aborder l'incertitude économique issue de la volatilité des revenus de chaque ménage. Trois sources d'incertitude seront ainsi identifiées et donneront chacune lieu à des recommandations de politique économique spécifique.

Soit:

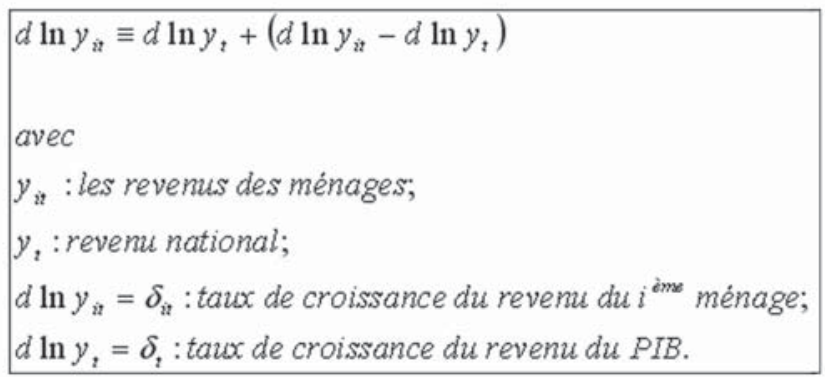

Soit le taux de croissance du revenu du ménage par rapport à la moyenne nationale défini par :

$$
\left(d \ln y_{i t}-d \ln y_{z}\right)=\rho_{i t}
$$

La volatilité de la croissance du revenu du i ìme ménage peut ainsi se décomposer en trois termes : 


$$
\begin{aligned}
& \sigma_{i}^{2}=\sigma_{i}^{2}+\sigma_{p}^{2}+2 \operatorname{cov}\left(\rho_{s}, \delta_{i}\right) ; \\
& \text { avec } \\
& \sigma_{\tilde{s}}^{2} \text { : volatilité de l'économie nationale; } \\
& \sigma_{\rho}^{2} \text { : volatilitédes revenus relatifs du ménage; } \\
& \operatorname{cov}\left(a_{n}, \delta_{i}\right) \text { : covarianceentre les toux de croissance du PIBet } \\
& \text { toux de croissance des revenus relatifs de chaqque ménage. }
\end{aligned}
$$

Ce type d'analyse basée sur des données micro-économiques de salaires issues d'enquêtes individuelles est difficile à mener. Aussi, $\mathrm{D}$. Rodrik se propose-t-il de passer à une étude avec des données de salaires réels moyens. Cela suppose la détermination d'un "salarié ou ménage moyen" dont on considérera la rémunération pour ensuite effectuer la décomposition proposée par l'auteur. Or, comme nous l'avons vu en étudiant le marché du travail du Mercosur, le concept même de salarié "moyen" qui plus est du secteur formel est difficile à défendre comme étant représentatif dans le pays comme le Brésil et le Paraguay mais désormais également en Argentine et en Uruguay.

Ces limites sont essentielles à souligner avant que l'on procède à ces tests avec des données de salaires réels moyens mais n'enlève en rien l'intérêt que cette étude peut représenter et les informations précieuses que celle-ci donne sur les décisions de politique économique à prendre pour être les plus efficaces.

Afin de mettre en pratique cette décomposition, D. Rodrik propose de se livrer au même exercice mais à partir d'un ménage moyen dont le salaire sera le salaire réel moyen. Ces données sont plus aisément disponibles et nous utiliserons les séries chronologiques exposées plus haut pour ces calculs. La décomposition s'écrit alors :

$$
\sigma_{v}^{2}=\sigma_{\delta}^{2}+\sigma_{\rho}^{2}+2 \operatorname{cov}(\rho, \delta)
$$

avec

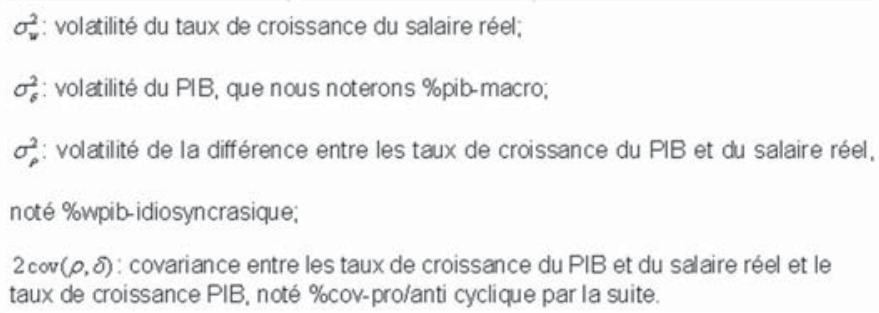


D. Rodrik présente les résultats pour quatre pays (Chili, Mexique, Pérou, et Venezuela) en décomposant la volatilité du salaire réel moyen lors de deux sous-périodes : l'une au cours des années 1980 et l'autre au cours des premières années de la décennie 1990.

Pour chaque élément de la décomposition et pour chaque sous-période, il calcule la décomposition et exprime la valeur de chacun des composants en pourcentage du total de la volatilité du salaire réel. Nous procéderons à partir de la même méthodologie en tentant de distinguer deux périodes caractéristiques des années 1990 pour les quatre pays du Mercosur.

Dans la majorité des cas, les données sont annuelles et la volatilité est calculée sur deux ou trois périodes (en fonction des pays et de la disponibilité des données). Pour l'Argentine et le Brésil, disposant de données trimestrielles pour les séries du taux de croissance du PIB réel et du salaire réel, celles-ci seront mobilisées pour pallier aux éventuels manques des données annuelles.

Les résultats sont synthétisés dans les graphiques ci-dessous qui indiquent pour chaque pays, et pour chaque période, la décomposition en pourcentage de la volatilité du salaire réel moyen en trois termes:

- un terme qui capte les perturbations macro-économiques (\%pib-macro);

- un terme qui capte les perturbations idiosyncrasiques c'est-à-dire propres du ménage ou travailleur qui perçoit le salaire moyen (\%wpib-idiosyncrasique);

- un terme qui prend en compte la covariance entre les taux de croissance du PIB et l'écart entre le taux de croissance du salaire et celui du PIB (\%cov-pro/anti cyclique). Ce terme est positif si le taux de croissance du salaire réel est pro-cyclique c'est-à-dire si le salaire réel augmente plus que la moyenne en période de croissance mais ralentit plus que la moyenne également en période de ralentissement. Si ce terme est négatif alors l'évolution du taux de salaire sera contra-cyclique .

La somme de ces trois termes (qui expriment pour chaque période et chaque pays, le pourcentage de la volatilité totale des salaires). La somme doit donc être égale à $1(100 \%)^{9}$.

\footnotetext{
${ }^{8}$ Dans une analyse à partir de données individuelles, l'intérêt et la justification analytique de ce terme estévidente. Il permet de distinguer différents types de ménages (riche, pauvres, indigents, etc.) et de confirme les analyses de N. Lustig (1999) concernant la vulnérabilité des ménages pauvres aux chocs macro-économiques. Néanmoins, en données macro-économiques, ce terme n'indique plus que le caractère pro-cylique ou contra-cyclique de l'évolution du salaire du travailleur "moyen".

9 Commenous le verrons dans les conclusions, les résultats que nous présentons présentent des sommes qui dépassent parfois l'unitéet qui sontl'expression non pas d'erreur de calcul mais de limites intrinsèques àce type de décomposition etcalculs.
} 


\subsubsection{Les résultats de la décomposition de la volatilité des salaires réels:}

Les résultats de ces décompositions sont présentés dans les quatre graphiques suivants:

\section{Graphique 6}

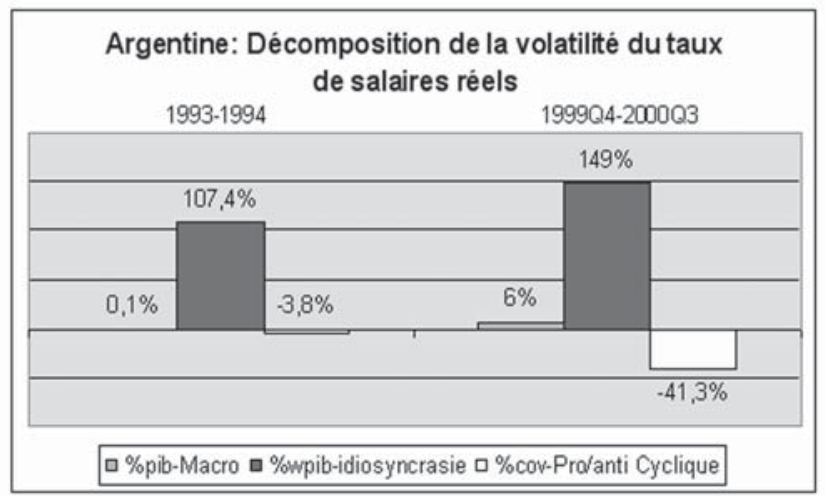

\section{Source: Calculs de l'auteur.}

La décomposition de la volatilité du taux de croissance du salaire réel moyen est problématique dans le cas de l'Argentine. En effet, comme nous l'avons déjà évoqué, les salaires ont été bloqués durant une longue période durant les années 1990. Il n’est donc pas surprenant que dans les deux sous-périodes considérées (1993-1994 et 1999Q42000Q3), ce soit le terme \%wpib-idiosyncrasie (qui capte la volatilité de la différence entre le taux de salaire réel moyen et le taux de croissance du PIB réel de l'économie) qui explique le plus la volatilité du salaire réel moyen en Argentine. Le terme macro-économique (\%pib-Macro) est quasi nul durant la période 1993-1994 (0,1\%) mais augmente à $6 \%$ au cours de la période 1999-2000. Si la volatilité macro-économique importait peu durant la période du miracle argentin, elle refait son apparition au début de la longue et destructrice crise qui débute dès 1999-2000. Au cours des deux sous-périodes, le terme qui mesure la covariance entre l'écart taux de salaire et PIB d'une part et taux de croissance du PIB d'autre part est négatif (-3,8\% et $-41,3 \%$ respectivement). Les résultats soulignent le caractère contra-cyclique qui correspond au blocage et à la rigidité à la baisse mais aussi à la hausse des salaires réels.

La méthodologie de Rodrik nous conduit à penser que la mesure de politique la plus efficace se situe au niveau idiosyncrasique. Nous reviendrons dans la section suivante sur les commentaires des résultats. 


\section{Graphique 7}

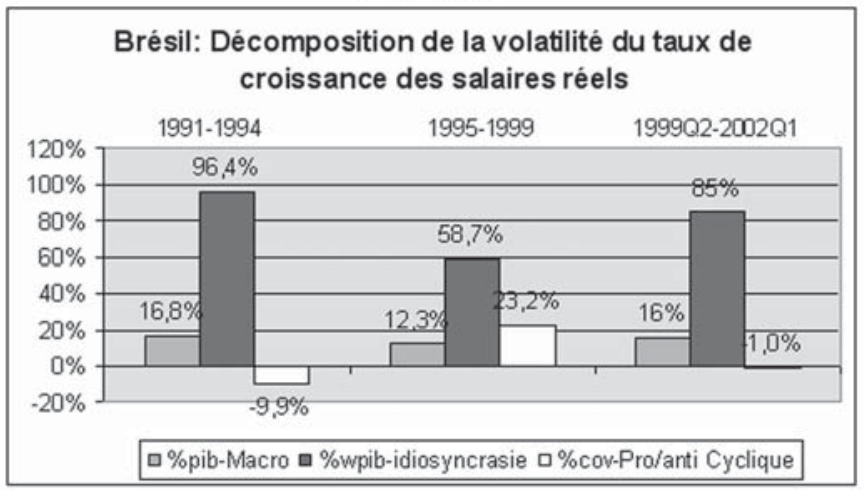

\section{Source: Elaboration de l'auteur.}

Au Brésil, trois sous-périodes peuvent être dégagées: 1991-1994 (hyperinflation), 1995-1999 (Plan real stabilisation macro-économique) et enfin 1999-2002 (en données trimestrielles). La décomposition de la volatilité du taux du salaire réel moyen indique dans les trois sous-périodes la prédominance du facteur idiosyncrasique (\%wpibidiosyncrasie). La période du Plan Real se singularise par rapport aux deux autres. En effet, la composante macro-économique de la volatilité du salaire diminue (de 16,8\% à $12,3 \%$ ), le terme idiosyncrasique également (de $96,4 \%$ à $58 \%$ ) mais surtout le troisième terme (\%cov) devient positif et indique le caractère pro-cyclique du salaire relatif (par rapport au PIB) du ménage. Le salaire a donc varié dans le même sens que le taux de croissance du PIB. On pourrait alors analyser ce résultat comme un effet de rattrapage des salaires en période de croissance économique. Néanmoins, les données dont nous disposons (en trimestrielles) et certaines analyses de la CEPAL (J. Katz), nous invitent à analyser le caractère procyclique comme une modification de la structure productive et donc du marché du travail. Le fait de mobiliser des données moyennes rend difficile l'analyse de cette modification de la structure productive sectorielle puisque l'on ne dispose que d'un seul indice de salaire moyen ${ }^{10}$.

Les politiques les plus efficaces sont donc celles qui garantiront sous forme propres à chaque travailleur moyen une certain stabilité du salaire du ménage par rapport à l'évo-

\footnotetext{
${ }^{10} \mathrm{D}$. Rodrik reviendra sur ce point sous forme de limite à la décomposition avec des données moyennes.
} 
lution du PIB. Les politiques macro-économiques auront également des conséquences sur la volatilité du salaire réel. Le maintien de l'idiosyncrasie des ménages s'interprète selon nous comme la persistance des inégalités dans le pays et même leur consolidation.

\section{Graphique 8}

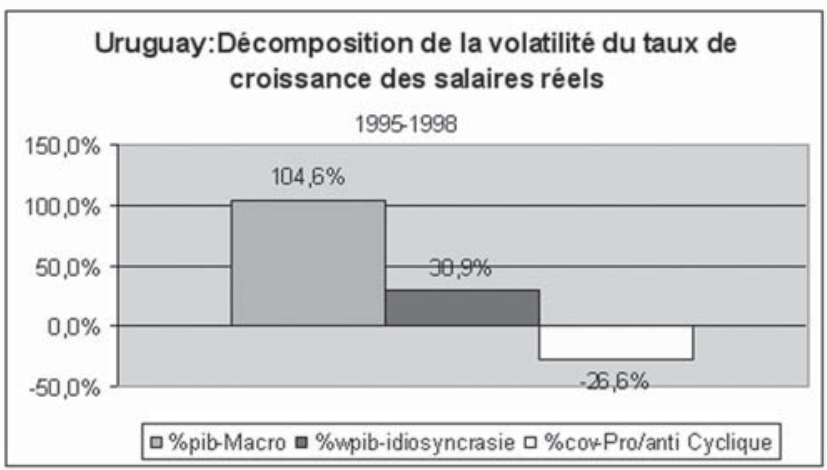

Source: Elaboration de l'auteur.

La seule phase qui a donné des résultats exploitables concerne, pour l'Uruguay, les années 1995-1998. Dans ce cas , le terme macro-économique est prédominant (104\%) contre seulement $30,9 \%$ pour le temre micro-économique et $-26,6 \%$ pour le terme anticyclique. Les politiques macro-économiques sont donc susceptibles d'amener des résultats plus efficaces. En l'absence d'autre période de comparaison l'intérêt est quelque peu limité.

\section{Graphique 9}

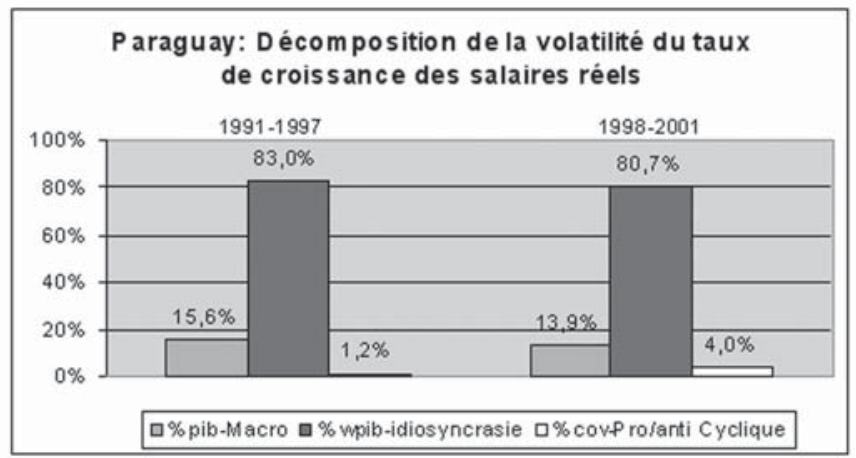

Source: Elaboration de l'auteur. 
Le Paraguay connaît une décomposition relativement semblable dans la période 1991-1997 et 1998-2002. Le terme idiosyncrasique domine largement les autres et le terme de covariance est positif (pro-cyclique).

\subsubsection{Commentaires des résultats et implications de politiques économiques:}

A partir de la distinction des trois sources de la volatilité de salaires, les résultats indique quel type de politique sera la plus efficace pour réduire la volatilité du salaire réel moyen :

- Si la cause est principalement macro-économique (\%pib-macro) alors les politiques macro-économiques de stabilisation pour faire baisser le risque de volatilité du revenu des ménages ;

- Si la cause est principalement idiosyncrasique (\%wpib-idiosyncrasie) alors D. Rodrik propose l'établissement d'une assurance (publique ou privée). Selon nous, ces politiques devraient prendre la forme de luttes contre les inégalités et la vulnérabilité face aux crises. Il s'agirait d'un partage de la croissance plus équitable entre capital et travail et de politiques contra-cycliques en période de récession pour soutenir les salaires et la demande qui en découle. Nous privilégions donc une optique de demande effective et de soutien à la demande du marché interne et régional (Mercosur).

- Si le terme exprimant la covariance est la cause principale (\%cov-Pro/anti cyclique) alors une politique de transfert s'avérerait, selon D. Rodrik, la plus efficace. L'identification des ménages permettrait que les mécanismes de transferts soient bien ciblés. Il importe, comme le souligne N. Lustig (1999), que ces politiques de survie des indigents et des pauvres ne soient pas soumises aux crises et bénéficient d'un statut hiérarchiquement supérieur dans les priorités des politiques publiques ${ }^{11}$.

En réalité comme nous allons le voir, il s'agira le plus souvent de trouver le bon équilibre entre ces trois types de politiques.

Létude de la décomposition de la volatilité du salaire réel nous amène à formuler plusieurs remarques:

Si l'objet et les préoccupations liés à la décomposition de la volatilité du salaire sont pertinents il n'en reste pas moins que les résultats et les calculs ne sont pas toujours satisfaisants. En effet, la somme des trois composants de la volatilité du salaire n'est pas toujours égale à la volatilité du salaire (la somme est parfois légèrement supérieure ou

\footnotetext{
${ }^{11}$ Sur ce sujet voir (Marques-Pereira, 2003) et (Lautier, 2003) et leur proposition de réelle séparation entre le budget social de la Nation et le budget général de la Nation.
} 
inférieure à 100\%). Ceci ne modifie cependant pas les résultats et ne joue que sur la marge des trois éléments (sans affecter le sens et le signe des résultats).

D. Rodrik souligne lui-même que le fait de passer à l'analyse par le salaire moyen réel limite les interprétations du modèle et de la décomposition ${ }^{12}$. Des analyses ${ }^{13}$ ont par ailleurs montré que les années 1990 ont vu l'accroissement de l'hétérogénéité productive et sociale en Amérique latine (travail qualifié en hausse et travail non-qualifié également en hausse mais avec des salaires bloqués). L’analyse avec des données moyennes constitue donc une limite importante de ce type d'étude.

De plus, cet indicateur du revenu ne prend en considération que le secteur formel. Or on a vu que le secteur informel est presque “institutionnalisé " ", dans les pays du Cône Sud. Au final, cette étude se veut un guide illustratif sur l'expérience d'un travailleur moyen ${ }^{15}$.

Il importe d'analyser les différences entre les décompositions entre période d'un point de vue dynamique. Si le facteur macro-économique était le principal élément de la volatilité des salaires dans une première sous-période alors les modifications que cela aura sur la structure des salaires s'accumulent dans la période suivante. Ainsi, les personnes vulnérables sont les plus touchées dans la première période (hyperinflation) alors leur situation sera d'autant plus difficile après un épisode de crise. Certains économistes (N. Lustig) soutiennent l'établissement de garanties concernant les programmes de soutien en période de crise.

\section{Conclusion}

En conclusion de cette analyse de la décomposition de la volatilité du salaire moyen, il apparaît que le deuxième terme (\%wpib-idiosyncrasie) est celui qui explique dans la plus

\footnotetext{
${ }^{12}$ "Le fait de baser les calculs sur le salaire moyen de toute l'économie joue contre la finalité de la méthodologie de cette décomposition puisque cela occulte la distinction entre le risque idiosyncrastique et macro-économique. L'incidence du risque entre travailleurs est supprimé." (Rodrik, 2001, p. 17)

${ }^{13}$ Voir (Pizarro,2001), (Dedecca, 2001) et (Katz, 2001)

${ }^{14}$ Voir (Lautier, 2003): "On revient à la question évoquée plus haut de «l'autonomie du politique». Il est illusoire d'espérer un consensus préalable de l'ensemble de la population pour mettre en œuvre ces propositions, de même que le démantèlement des politiques sociales et l'évolution de la répartition du revenu qui a fait de l'Amérique latine le continent le plus inégalitaire du monde n'ont pas non plus reposé sur le consensus. Il est évident qu'un tel ensemble de propositions ne peut être mis en œuvre que dans des circonstances politiques particulières. La faillite amorcée des politiques néo-libérales en Amérique latine en crée en partie les conditions; certains événements électoraux récents également. Il est donc temps de lancer le débat.” (p. 81).

${ }^{15}$ Au Brésil, le différentiel de rémunération entre secteur formel et informel (sans couverture administrative ou à son compte) s'élevait en moyenne sur la décennie 1990 à $25 \%$ environ avec de nombreuses disparités régionales et sectorielles. Voir (IPEA-MTE, 2003, p. 46-47) et (CEP, 2003) pour une distinction entre secteurs (2 digits) en Argentine.
} 
large mesure la volatilité des salaires. Les politiques d'assurance dont par D. Rodrik comme étant les plus efficaces dans ce cas (sans toutefois les spécifier) devrait selon nous garantir que les fruits de la croissance (quand elle est présente) soient mieux distribués et notamment à ceux qui la créent c'est-à-dire les salariés. En période de récession, les politiques devraient viser à soutenir les salaires et veiller à ce qu'ils ne chutent pas trop afin de ne pas entamer encore plus la demande et la consommation. Il est clair que de telles mesures n'ont pas été prises et qu’elles supposent un changement de cap important mais selon nous nécessaire .

Cette étude s'inscrit dans un travail plus large qui met en résonance la volatilité macro-économique et la vulnérabilité sociale. Dans (Saludjian, 2004a), nous avons montré que le régime de croissance qui a prévalu dans le Mercosur n'a pas profité aux salariés qui n'ont pas vu leurs salaires suivre les évolutions positives de la croissance du PIB et de la productivité du travail. En ce sens, elle est une croissance excluante. Les réformes libérales et la non-activation du filet de sécurité régional du Mercosur ont contribué à accroître l'insécurité économique et ce, à travers la volatilité et les crises systémiques qui ponctuent la période 1991-2003.

Létude au niveau global de la volatilité et du marché du travail (et des salaires) cache la spécificité des différents secteurs productifs et des caractéristiques de la main d'œuvre qui y travaille. Le mode d'insertion dans l'économie mondiale et les marchandises qui vont y être vendues, le caractère qualifié ou non-qualifié de la main d'œuvre qui vont les produire influencent beaucoup les performances macro-économiques mais aussi dans les conditions sociales des travailleurs dans les différents secteurs.

Ainsi à partir de (Lautier , 2003), (Salama, 2003) et (Saludjian, 2004a), nous pouvons dire que dans tous les pays du Mercosur, la vulnérabilité sociale s'est accrue montrant l'inefficacité du Mercosur à constituer un filet de sécurité régional capable de pallier la libéralisation et l'ouverture.

On peut alors conclure que si les années 1980 ont constitué la "décennie perdue" selon l'expression consacrée par la CEPAL, les années 1990 ont été celles de la "décennie perdue au niveau social". Comme le souligne la CEPAL, "la vulnérabilité sociale (due à la qualité de l'emploi, du capital humain, des relations sociales et de la rareté mais aussi de la perte de capital dans les micro et petites entreprises) est devenue le trait essentiel de la société latino-américaine de ce début de siècle”. (CEPAL, 2001b, p.51)

\footnotetext{
${ }^{16}$ Voir (Lautier, 2003, p. 81).
} 


\section{BIBLIOGRAPHIE}

DEDECCA, C. “Mercosul: Limites e Perspectivas", in TEM, 2001, ouvrage cité.

INDEC, 2003, site internet www.indec.mecon.ar.

IPEADATA, 2003, site internet. www.ipea.org.br

KATZ J. "Structural reforms, productivity and technological change in Latin America”, LIBROS DE LA CEPAL. Santiago du Chili, n. 64, 2001, 143 p.

LAUTIER B. Une protection sociale mutualiste et universelle en Amérique Latine: la condition de l'efficacité de la lutte contre la pauvreté, in SALAMA P., MARQUESPEREIRA J. et LAUTIER B., article cité, 2003.

LUSTIG N. "Crises and the poor: socially responsible macroeconomics”, Presidential Address Sept. Washington DC, BID, 1999.

MARQUES-PEREIRA J. "La crédibilité d'une politique anti-cyclique en Amérique Latine: Les imperfections des marchés financiers à l'aune d'un déséquilibre externe structurel”, in SALAMA P. et ali , article cité, 2003.

MORTIMORE M., VERGARA S. et KATZ J. "La competitividad internacional y desarrollo nacional: implicancias para la política de Inversión Extranjera Directa (IED) en América Latina”. Santiago du Chili, Serie productivo, n. 107, 2001.

MTE (Ministerio do Travalho e Emprego) Observatório do Mercado de Travalho do Mercosul, Boletim da Seção Brasileira. Brasília, Ano 1, n. 1, Juin, MTE, 2001.

PIZARRO R. "La vulnerabilidad social y sus desafios: una mirada desde América Latina”, Serie Estudios estadisticos y prospectivos. División de estadistica y Proyecciones Economicas de la CEPAL, Santiago du Chili, n. 6, fev, 2001.

RODRIK D. "Por que hay tanta inseguridad económica en América Latina?" Revista de la CEPAL. Santiago du Chili, n. 73, avril, 2001.

SALAMA P., J. MARQUES-PEREIRA et LAUTIER, B. "Régime de croissance, vulnérabilité financière et protection sociale en Amérique latine", serie Financiamiento del Desarrollo, site internet, Santiago du Chili, 2003.

SALAMA P. "Globalisation, inégalités territoriales et salariales", in Revue Tiers-Monde. Paris, PUF, t. XXXIX, n. 154, 1999.

SALAMA P., "La financiarisation excluante: les leçons des économies latino-américaines" in CHESNAIS F. (Coord.), La Globalisation financière: Génèse, coûts et enjeux. Paris: La Découverte-Syros, 1996.

SALUDJIAN A. Una crítica al Mercosur liberal y financiarizado: Para un cambio hacia la homogeneidad estructural y social. Argentine: Editions Libros del Zorzal, BuenosAires, thèse publiée et traduite en espagnol, (à paraître en Novembre), $2004 a$. 
SALUDJIAN A., Trajectoires de croissance, régime de change et volatilité dans le Mercosur: Quelques éléments d'analyse empirique, in Revue Tiers-Monde. Paris, Ed. PUF, n. 179, juillet-sept, 2004b, pp. 595-615.

STALLINGS B. et PERES W. Crecimiento, empleo y equidad, El impacto de las reformas económicas en América Latina y el Caribe, FCE-CEPAL, Santiago du Chili, 2000.

WILLIAMSON J. et P-P Kuczynski (Coord.) After the Washington Consensus, Restarting Growth and Reform in Latin America, Ed. Institute for International Economics. Washington D. C. mars, 2003.

WOOD A. "Openness and wage inequality in developing countries: the latin america challenge to east asian conventional wisdom", in World Bank Economic Review, v. 11, n. 1, Washington D.C., 1997. 